\title{
Chronic diarrhea because of villous atrophy unrelated to celiac disease
}

A 71-year-old woman was admitted with a 7-month history of watery diarrhea, which had led to an unintentional $30 \mathrm{~kg}$ (27\%) of weight loss and admittance to the intensive care unit (ICU) on two separate occasions as a result of dehydration. Endoscopy and video capsule evaluation revealed villous atrophy of the entire small bowel, with fissuring, nodularity, and loss of folds, as shown for both duodenum ( $\bullet$ Fig. 1 a) and ileum ( $\bullet$ Fig.1b). The mucosa appeared fragile, with ulcers after biopsies ( $\bullet$ Fig. 1 c). Histology confirmed complete villous atrophy and showed lengthened regenerative crypts, only a few intraepithelial lymphocytes, and thickening of the basal membrane in both proximal ( $\bullet$ Fig. 2) and distal small-bowel biopsies. Colonoscopy revealed a pale and edematous mucosa with superficial ulcerations, more pronounced distally ( Fig.3). Microscopic evaluation showed subtle inflammation in colon biopsies with focal erosion, a focally thickened basal membrane, and some apoptotic cells in the epithelium ( $\bullet$ Fig. 4 a, b). Infectious, ischemic, and malignant disorders were excluded. Serum anti-tTG IgA and anti-gliadin IgG were negative during and after gluten exposure, ruling out celiac disease. The clinical presentation and diagnostic findings were most compatible with adult-onset autoimmune enteropathy [1], affecting an extensive part of the digestive tract (stomach to rectum). Immunosuppressive therapy was started; however high dose prednisolone, increasing doses of azathioprine, and immunoglobulins failed to induce any clinical response. The patient continued to produce voluminous diarrhea; however 3 weeks after starting therapy with oral budesonide $(3 \times 3 \mathrm{mg}$ daily, pulverized in the morning, granules at noon, capsule at night) [1], the patient recovered, with formed stools, clinical improvement, and weight gain. Duodenal biopsies revealed completely restored villous architecture

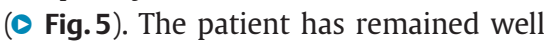
for 20 months of follow-up.

Thus, autoimmune enteropathy should be considered after exclusion of celiac disease when severe diarrhea is associated with villous atrophy. Topical immunosuppressive treatment should be applied.

Endoscopy_UCTN_Code_CCL_1AC_2AD

\section{Competing interests: None}

\section{Anja Ursula van Lent ${ }^{1}$, R. Bart Takkenberg ${ }^{1}$, Susanne van Eeden ${ }^{2}$, Chris Mulder $^{3}$, Ulrich Beuers ${ }^{1}$}

${ }^{1}$ Department of Gastroenterology and Hepatology, Academic Medical Center, University of Amsterdam, Amsterdam, The Netherlands

2 Department of Pathology, Academic Medical Center, University of Amsterdam, Amsterdam, The Netherlands ${ }^{3}$ Department of Gastroenterology and Hepatology, VU University Medical Center, University of Amsterdam, Amsterdam, The Netherlands
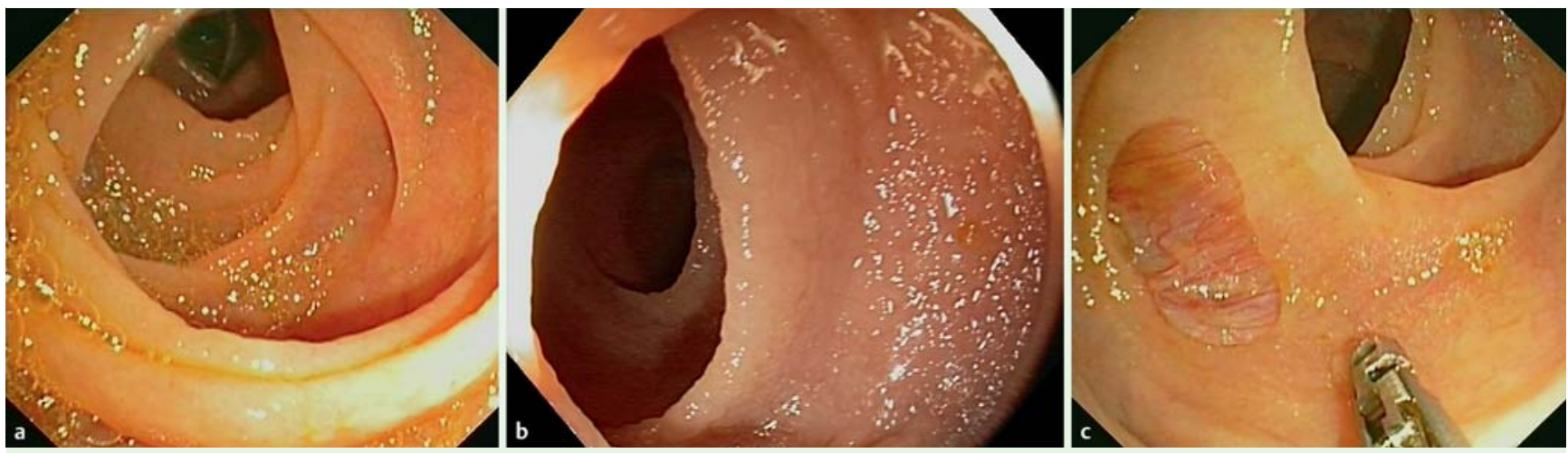

Fig. 1 A 71-year-old woman was admitted with a 7-month history of watery diarrhea and weight loss. Endoscopic images showing: a villous atrophy in the duodenum; $\mathbf{b}$ villous atrophy in the ileum; $\mathbf{c}$ ulcers after biopsies in the duodenum.

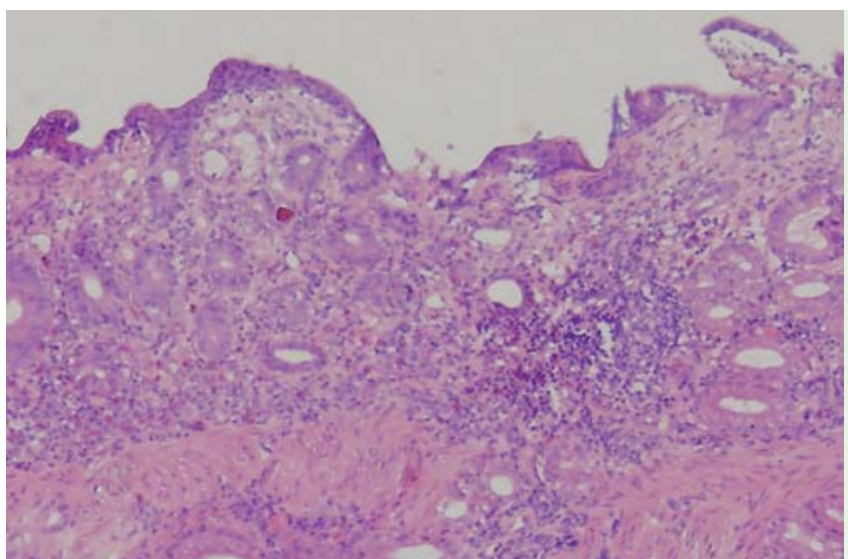

Fig. 2 Proximal smallbowel biopsy (hematoxylin and eosin (H\&E) stain) of duodenal mucosa demonstrating severe villous atrophy, some inflammation, and thickening of the basal membrane.

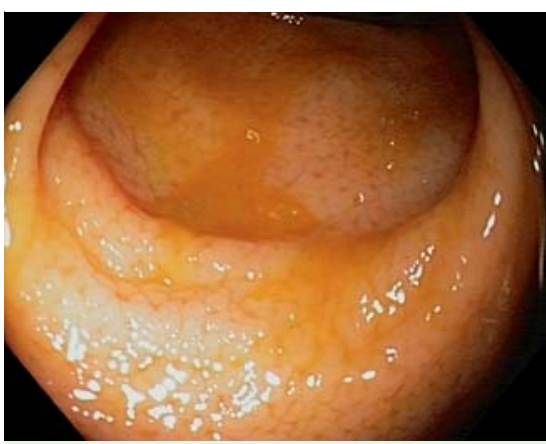

Fig. 3 Colonoscopy of the distal colon showing pale and edematous mucosa with superficial ulcerations. 


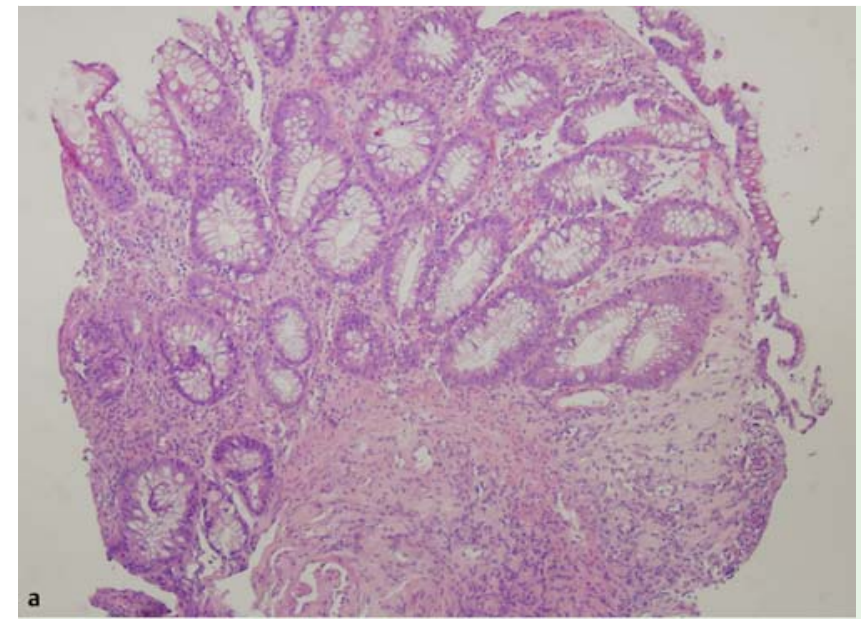

Fig. 4 Colonic mucosa (H\&E staining) showing: a focal erosion with homogenization of the lamina propria; $\mathbf{b}$ some apoptotic cells (arrows) in the epithelium.

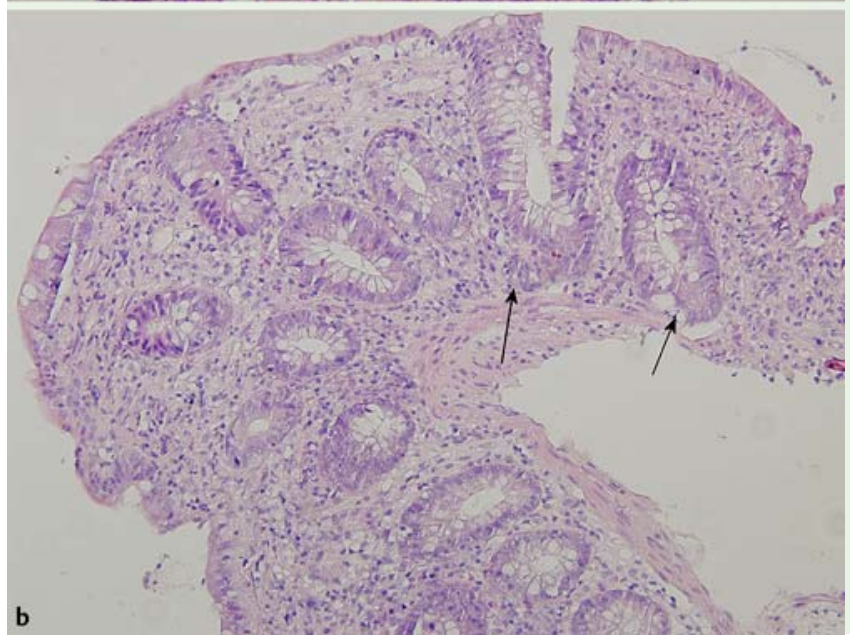

Bibliography

DOI http://dx.doi.org/

10.1055/s-0034-1391247

Endoscopy 2015; 47: E71-E72

(c) Georg Thieme Verlag KG

Stuttgart . New York

ISSN 0013-726X
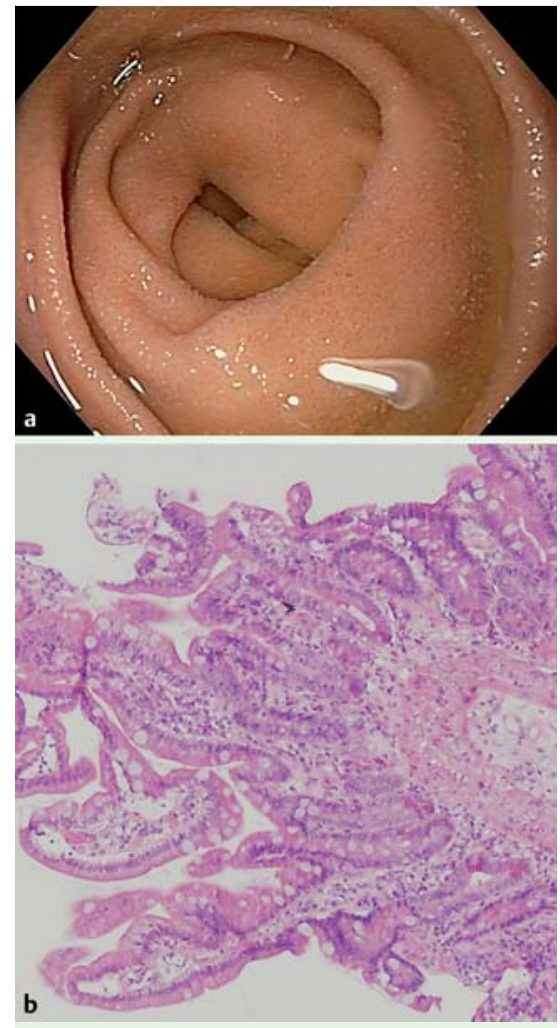

Fig. 5 Restored villous architecture after topical budesonide treatment: a endoscopic view; b microscopic view.

\section{Corresponding author}

Anja Ursula van Lent, MD, PhD

Department of Gastroenterology and Hepatology

Academic Medical Center

University of Amsterdam

P.O. Box 22700

1100 DE Amsterdam

The Netherlands

Fax: +31-20-5669608

a.u.vanlent@amc.uva.nl 